\title{
An unexpected role for angiotensin II in the link between dietary salt and proximal reabsorption
}

\author{
Scott C. Thomson, Aihua Deng, Lucinda Wead, Kerstin Richter, Roland C. Blantz, and Volker Vallon \\ Department of Medicine, Division of Nephrology — Hypertension, University of California, San Diego, and VA San Diego Healthcare System, \\ San Diego, California, USA.
}

\begin{abstract}
We set out to confirm the long-held, but untested, assumption that dietary salt affects proximal reabsorption through reciprocal effects on the renin-angiotensin system in a way that facilitates salt homeostasis. Wistar rats were fed standard or high-salt diets for 7 days and then subjected to renal micropuncture for determination of single-nephron GFR (SNGFR) and proximal reabsorption. The tubuloglomerular feedback (TGF) system was used as a tool to manipulate SNGFR in order to distinguish primary changes in net proximal reabsorption (Jprox) from changes due to glomerulotubular balance. The influence of Ang II over Jprox was determined by the sensitivity of Jprox to the AT1 receptor antagonist, losartan. Plasma, whole kidneys, and fluid from midproximal tubules were assayed for Ang II content by radioimmunoassay. In rats on the standard diet, losartan reduced Jprox by $25 \%$ and reduced the maximum range of the TGF response by $50 \%$. The highsalt diet suppressed plasma and whole-kidney Ang II levels. But the high-salt diet failed to reduce the impact of losartan on Jprox or the TGF response and actually caused tubular fluid Ang II content to increase. The persistent effect of Ang II on Jprox prevented a major rise in late proximal flow rate in response to the high-salt diet. These observations challenge the traditional model and indicate that the role of proximal tubular Ang II in salt-replete rats is to stabilize nephron function rather than to contribute to salt homeostasis.
\end{abstract}

\section{Introduction}

The archetypal renin-angiotensin system (RAS) stabilizes blood pressure by negative feedback control of both peripheral vascular resistance and total body salt (1). Control of total body salt is mediated by pro-reabsorptive effects of Ang II and aldosterone in the proximal and distal nephrons, respectively. The present study concerns the role of Ang II in proximal reabsorption. It is well established that reducing the total body salt activates the systemic RAS, and it is reasonable to assume that Ang II in and around the proximal tubule correlates with Ang II elsewhere in the body and thereby contributes to salt homeostasis.

If it is true that Ang II contributes to salt homeostasis by affecting proximal reabsorption, and if the assumption is correct that Ang II in proximal tubular fluid correlates with systemic Ang II, then a change in proximal tubular Ang II must lead to a parallel change in proximal reabsorption. However, previous data from several investigators suggest that a self-contained tubular angiotensin system may operate independently of the systemic RAS (2-4). For example, single-nephron hyperfiltration may cause the proximal tubule to produce angiotensinogen (5), while acute volume expansion suppresses Ang II in the whole kidney but not in proximal tubular fluid (6). These reports provide a reason to question the traditional paradigm for salt homeostasis, since a pro-reabsorptive proximal tubular Ang II system must be antihomeostatic if it is provoked by an increase in total body salt.

Nonstandard abbreviations used: ATF, artificial tubular fluid; FPR, fractional proximal reabsorption; GTB, glomerulotubular balance; Jprox, proximal reabsorption; RAS, renin-angiotensin system; SNGFR, single-nephron GFR; VLP, late proximal flow. Conflict of interest: The authors have declared that no conflict of interest exists. Citation for this article: J. Clin. Invest. 116:1110-1116 (2006). doi:10.1172/JCI26092.
In the present study, we set out to confirm the long-held, but untested, assumptions regarding Ang II, proximal reabsorption, and salt homeostasis. We measured proximal tubular fluid Ang II concentration and proximal reabsorption in rats fed standard and high-salt diets and subjected to acute AT1 receptor blockade. We report that under standard diet, the net effect of endogenous Ang II was pro-reabsorptive as suspected. However, Ang II-dependent proximal reabsorption was not lessened by the high-salt diet. Instead, the proximal tubular effect of Ang II persisted and stabilized late proximal flow (VLP) during the transition from the standard to the high-salt diet. Furthermore, feeding a high-salt diet had the unexpected effect of increasing Ang II content of the proximal tubule fluid. These observations challenge the traditional model and reveal that the role of proximal tubular Ang II is to stabilize nephron function rather than contribute to salt homeostasis.

\section{Results}

Clearance and micropuncture data were obtained before and during acute AT1 receptor blockade with losartan in rats fed a standard diet and in rats fed a high-salt diet for 1 week. A standard surgical preparation was employed for micropuncture with Inactin anesthesia (Research Biochemicals International). Micropuncture collections were made from the late proximal tubule with and without activating tubuloglomerular feedback (TGF) in order to render singlenephron GFR (SNGFR) an independent variable for the purpose of detecting primary effects on proximal reabsorption. A primary effect on tubular reabsorption is a change in reabsorption not mediated by glomerulotubular balance (GTB). Ang II was measured by radioimmunoassay in plasma, whole kidneys, and proximal tubular fluid. See Methods for a detailed description of the procedures.

Blood pressure and GFR. Losartan reduced blood pressure in rats fed the high-salt diet for 1 week and in rats fed standard diets. 
Table 1

Blood pressure and GFR

$\begin{array}{cccccc} & \begin{array}{c}\text { Body } \\ \text { wt }\end{array} & \begin{array}{c}\text { BP } \\ (\mathbf{m m H g})\end{array} & \begin{array}{c}\text { BP during } \\ \text { losartan } \\ (\mathbf{m m H g})\end{array} & \begin{array}{c}\text { GFR } \\ (\mathbf{m l} / \mathbf{m i n})\end{array} & \begin{array}{c}\text { GFR during } \\ \text { losartan } \\ (\mathbf{m l} / \mathbf{m i n})\end{array} \\ \text { Standard } & 316 \pm 10 & 124 \pm 4 & 107 \pm 5^{\mathrm{A}} & 2.4 \pm 0.1 & 2.3 \pm 0.4 \\ \text { High-salt } & 332 \pm 7 & 129 \pm 10 & 118 \pm 9^{\mathrm{A}} & 2.9 \pm 0.3 & 2.7 \pm 0.4\end{array}$

Values are mean \pm SEM. BP, mean arterial blood pressure. ${ }^{A} P<0.05$ versus pre-losartan.

As might be expected, there were tendencies toward higher blood pressure and a lesser effect of losartan on blood pressure in rats fed the high-salt diet, but these were not significant. There was also a nonsignificant tendency for 2-kidney GFR to be greater in rats fed the high-salt diet. GFR was essentially unaffected by losartan in both groups (Table 1).

SNGFR and proximal reabsorption. Micropuncture data were obtained from 13 rats fed the standard diet and from 10 rats fed the high-salt diet. The mean body weight was not different between rats fed standard and high-salt diets. Within each group, much of the variance in SNGFR was attributable to body weight $(r=0.5 ; P=0.004$ for standard and $P<0.001$ for high-salt). Therefore, analysis of variance for the effects of salt and losartan included body weight as a covariate. Paired collections were made such that data were available for each nephron during maximum activation of TGF and in the absence of any TGF stimulus. SNGFR data are shown in Table 2. Rats fed the high-salt diet manifested higher SNGFR regardless of the state of TGF activation or Ang II AT1 receptor blockade. The magnitude of this effect was proportional to the apparent effect of dietary salt on whole-kidney GFR. However, due to lesser variance for the micropuncture results, the effect of salt on SNGFR was statistically significant $(P<0.0005)$, whereas the effect on GFR was not (see above). Notably, the high-salt diet did not alter the maximum TGF response (Table 2). Losartan had no effect on SNGFR in the absence of a TGF stimulus. This is consistent with several prior micropuncture studies from our lab showing that glomerular hemodynamics, and hence the early peritubular capillary Starling forces, are unaffected by angiotensin blockade in salt-replete rats (7-9). This obviated concern over peritubular capillary physical factors originating from the glomerulus when analyzing the proximal diuretic effects of losartan. However, losartan did blunt the maximum TGF response by about half $(P=0.002)$. This effect of losartan was independent of dietary salt (Table 2).

The effects of dietary salt and losartan on proximal reabsorption are shown in Table 3. Dietary salt had no effect on fractional proximal reabsorption (FPR), but net proximal reabsorption (Jprox) was greater in rats fed the high-salt diet. Actual VLP for a free-flowing nephron must lie somewhere between the values associated with minimal and maximal TGF activation. Both upper and lower bounds for VLP were increased by about $4 \mathrm{nl} / \mathrm{min}$ in rats fed the high-salt $\operatorname{diet}(P<0.05)$, but the range of VLP overlap between rats fed the standard and high-salt diets was much larger than this. Furthermore, under conditions of volume expansion, nephrons are expected to operate closer to the elbow of the TGF curve (10). Therefore, it is likely that the high-salt diet caused ambient VLP to increase by some amount less than $4 \mathrm{nl} / \mathrm{min}$. We also do not know how much of the difference in tubular flow was dissipated by greater reabsorption between the collection site and the early distal nephron. We previously confirmed that Sprague-Dawley rats on a high-salt diet can achieve an appropriate increase in salt excretion without increasing the amount delivered to the early distal nephrons (11). VLP was not measured in those particular rats, and they did not hyperfilter.

Losartan decreased both FPR $(P<0.0005)$ and Jprox $(P<0.02)$. This effect was independent of dietary salt. Perfusing Henle's loop to activate TGF caused a greater relative decline in SNGFR than in proximal reabsorption. As a result, FPR was greater when SNGFR was least. This confirms prior reports demonstrating imperfect GTB in the proximal tubule $(11,12)$. There was no significant effect of dietary salt or losartan on GTB efficiency, although for no apparent reason all groups manifested somewhat less GTB efficiency than seen in other recent studies (11, 12). Losartan shifted the range of available VLP far enough upward to eliminate most of the overlap and effectively establish that VLP must have increased during losartan in free-flowing nephrons. The high-salt diet did not suppress this effect of losartan (Table 3).

The present experiments were designed so that SNGFR could be treated as an independent variable in order to test for primary differences in proximal reabsorption, where a primary difference is any change not due to GTB. The results of this analysis are depicted in Figure 1, which makes pairwise comparisons of proximal reabsorption. The first comparison, between rats fed standard and high-salt diets, shows that Jprox was greater in rats fed the highsalt diet (Figure 1A). While this might seem paradoxical, a similar tendency was previously noted in this same model of male Wistar rats (11) and also observed in female Wistar rats (our unpublished observations). The second comparison illustrates the primary inhibitory effect of losartan on proximal reabsorption in rats fed the standard diet (Figure 1B). Another comparison illustrates the response to losartan in rats fed the high-salt diet, showing that the inhibitory effect of losartan on proximal reabsorption persisted on a high-salt diet (Figure 1D). A final comparison illustrates what would have been the effect of a high-salt diet on proximal reabsorption had the high-salt diet suppressed the effects of tubular Ang II (Figure 1C). Additional comparisons are made in Figure 2, which illustrates that the persistence of tubular Ang II activity in

\section{Table 2}

SNGFR in standard and high-salt diets with and without TGF activation

\begin{tabular}{lrrr} 
& SNGFR $_{\mathbf{0}}$ & SNGFR $_{\mathbf{3 8}}$ & TGF range \\
Value $(\mathbf{n l} / \mathbf{m i n})^{\mathbf{A}}$ & & & \\
Standard $(n=37)$ & $29.8 \pm 1.5$ & $19.4 \pm 1.6$ & $10.4 \pm 1.4$ \\
Standard + losartan $(n=30)$ & $32.8 \pm 1.7$ & $27.6 \pm 1.8$ & $5.2 \pm 1.5$ \\
High-salt $(n=39)$ & $38.0 \pm 1.5$ & $25.5 \pm 1.6$ & $12.5 \pm 1.4$ \\
High-salt + losartan $(n=22)$ & $37.1 \pm 2.0$ & $30.9 \pm 2.2$ & $6.2 \pm 1.9$ \\
$\boldsymbol{P v}_{\text {value }}$ B & & & \\
Salt & $<0.001$ & 0.010 & 0.329 \\
Losartan & 0.530 & $<0.001$ & $<0.001$ \\
Salt $\times$ losartan & 0.259 & 0.468 & 0.716 \\
\hline
\end{tabular}

Subscripts indicate $\mathrm{nl} / \mathrm{min}$ rate of perfusion of Henle's loop with ATF. AMean \pm SEM determined by analysis of covariance. ${ }^{B} P$ values determined by 2 -way ANOVA. 
Table 3

Proximal reabsorption in standard and high-salt diets with and without TGF activation

\begin{tabular}{|c|c|c|c|c|c|c|}
\hline & Jprox $_{0}$ & Jprox $_{38}$ & $\mathrm{FPR}_{0}$ & $\mathrm{FPR}_{38}$ & $\mathrm{VLP}_{0}$ & $\mathrm{VLP}_{38}$ \\
\hline \multicolumn{7}{|l|}{ Value $(\mathrm{nl} / \mathrm{min})^{\mathrm{A}}$} \\
\hline Standard $(n=37)$ & $10.9 \pm 0.8$ & $10.3 \pm 0.9$ & $0.38 \pm 0.02$ & $0.53 \pm 0.03$ & $18.9 \pm 1.3$ & $9.1 \pm 1.3$ \\
\hline Standard + losartan $(n=30)$ & $9.7 \pm 0.9$ & $8.5 \pm 1.0$ & $0.31 \pm 0.03$ & $0.35 \pm 0.03$ & $23.1 \pm 1.4$ & $18.7 \pm 1.4$ \\
\hline High-salt $(n=39)$ & $14.1 \pm 0.8$ & $12.6 \pm 0.9$ & $0.38 \pm 0.02$ & $0.50 \pm 0.03$ & $23.8 \pm 1.3$ & $12.8 \pm 1.3$ \\
\hline High-salt + losartan $(n=22)$ & $10.3 \pm 1.1$ & $9.9 \pm 1.2$ & $0.27 \pm 0.03$ & $0.33 \pm 0.04$ & $26.8 \pm 1.8$ & $21.1 \pm 1.8$ \\
\hline \multicolumn{7}{|c|}{$P$ value ${ }^{B}$} \\
\hline Salt & 0.036 & 0.088 & 0.375 & 0.436 & 0.003 & 0.033 \\
\hline Losartan & 0.006 & 0.037 & 0.001 & $<0.001$ & 0.015 & $<0.001$ \\
\hline Salt $\times$ losartan & 0.155 & 0.520 & 0.498 & 0.959 & 0.683 & 0.628 \\
\hline
\end{tabular}

Subscripts indicate $\mathrm{nl} / \mathrm{min}$ rate of perfusion of Henle's loop with ATF. AMean \pm SEM determined by analysis of covariance. ${ }^{B} P$ values determined by 2-way ANOVA.

rats fed a high-salt diet reduced what would otherwise have been a much larger increase in VLP.

Dietary salt and Ang II content of plasma, whole kidneys, and proximal tubular fluid. As shown in Figure 3, the high-salt diet reduced Ang II concentration by $57 \%$ in plasma and $22 \%$ in the whole kidney $(P<0.05)$. In contrast, proximal tubular fluid from rats fed the high-salt diet contained significantly more Ang II than tubular fluid from rats fed the standard diet ( $P<0.05, n=5$ rats per group). The total amount of Ang II passing the midproximal tubule also appeared to be increased in high-salt nephrons ( $7 \pm 1$ and $12 \pm 2$ $\mathrm{fg} / \mathrm{min}$ in standard and high-salt nephrons, respectively; $P=0.06$ ).

\section{Discussion}

The present data contain several surprises. First, feeding rats with the high-salt diet for 1 week led to a net increase in proximal reabsorption. This is contrary to what might be expected based on the traditional archetype for salt homeostasis, but it is not unprecedented. We have previously witnessed accelerated kidney growth in rats fed the high-salt diet along with a tendency toward increased glomerular filtration and proximal reabsorption, which likely reflects the greater functioning tissue mass (ref. 11 and our unpublished observations). In the present experiments this effect achieved statistical significance where it previously has not, but in spite of this primary increase in proximal reabsorption, VLP was not reduced by the high-salt diet (Table 3 and Figure 2). Therefore, this effect of dietary salt on Jprox does not present a conundrum for salt balance.

Another surprise is that the range of the TGF response (e.g., the difference between SNGFR during minimum and maximum stimulation of TGF) was not suppressed by the high-salt diet. This would also contradict what many physiologists might expect, since according to common understanding of macula densa physiology,

\section{Figure 1}

Jprox as a function of SNGFR. In order to make SNGFR an independent variable, Henle's loop was perfused to alter the activity of tubuloglomerular feedback. Data points and error bars represent adjusted least-squares mean \pm SEM. The vertical distance between the solid and dashed lines reflect primary differences in proximal reabsorption. Losartan reduced Jprox regardless of dietary salt (B and D). The highsalt diet increased SNGFR and Jprox (A), but had tubular Ang II been suppressed by the high-salt diet, the increase in Jprox would not have occurred (A and C). $P<0.05$ for SNGFR $>26$ (A), SNGFR $<26$ (B), and all SNGFR (D). high dietary salt should reduce the activity of Ang II and increase the formation of macula densa nitric oxide. Ang II is the principal modulator known for strengthening the TGF response $(13,14)$, and macula densa nitric oxide is the principal modulator known for weakening the TGF response (15-18). Nonetheless, there is precedent for dietary salt to lack influence over the range of the TGF response evoked by perfusing Henle's loop with artificial tubular fluid (ATF) $(11,19)$. Furthermore, the present data regarding the TGF response are in keeping with expectations inasmuch as angiotensin receptor blockade reduced the range of the TGF response by about half (Table 2 and Figure 1).

A third surprise among the micropuncture data demands greater explanation. This involves endogenous Ang II as an effector of proximal reabsorption and its modulation by dietary salt. The reciprocal effects of dietary salt on plasma and kidney Ang II in the present studies were entirely expected, since we have previously documented that plasma and whole-kidney concentrations of Ang II increase during dietary salt restriction in rats (20), and others have confirmed this (21). Therefore, we surmised that Ang II would exert a lesser tonic influence over proximal reabsorption in rats fed a high-salt diet and that this would appear as a lesser decrease in proximal reabsorption during treatment with losartan. Indeed, losartan did reduce proximal reabsorption in rats on a standard diet, confirming that the net effect of endogenous Ang II is proreabsorptive. But contrary to our expectations, dietary salt load-
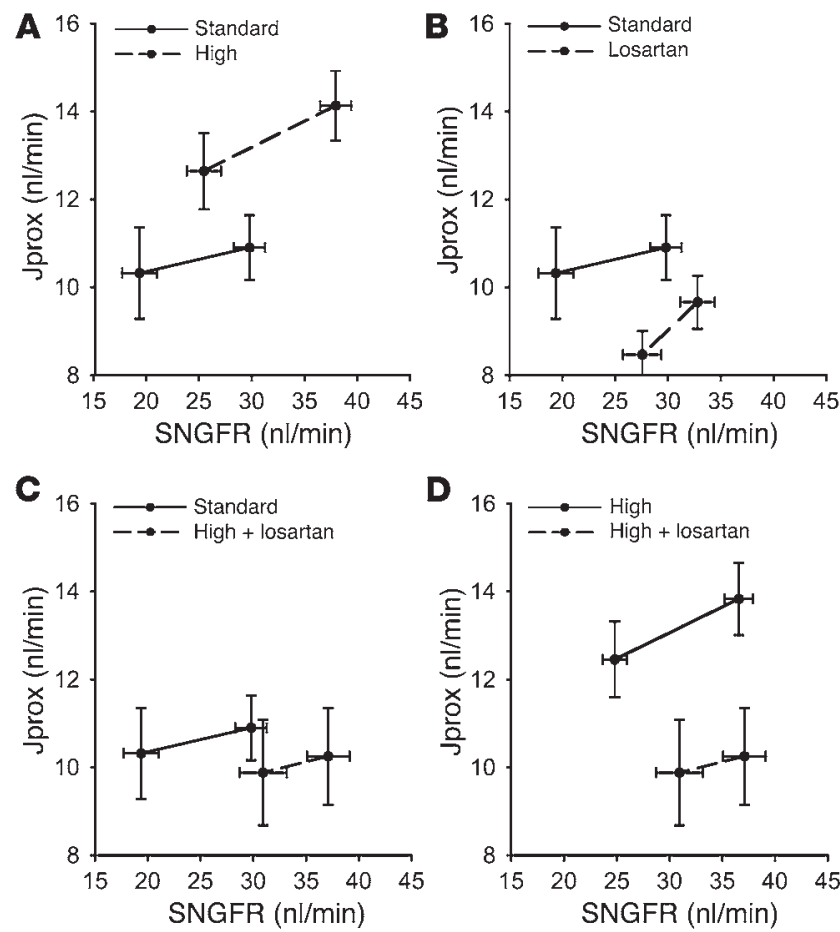


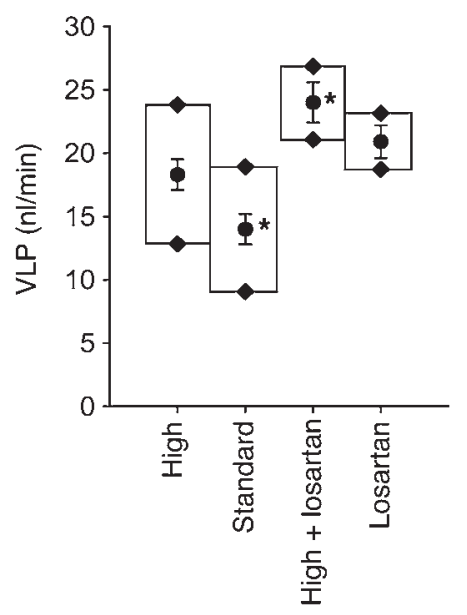

Figure 2

Range of possible VLPs. The upper and lower bounds of each box correspond to the values obtained at 0 and maximum TGF stimulation, respectively. The filled circle and error bars in the center of each box designate the mean \pm SEM obtained by averaging the upper and lower bounds for VLP in each nephron. This is where a typical nephron operates in the hydropenic rat. Nephrons in volume-expanded rats tend to operate closer to the elbow of the TGF response, so the value assigned to the high-salt diet likely overestimates the ambient VLP. The main point of this figure is to illustrate the major impact that the high-salt diet would exert on VLP if it succeeded in suppressing tubular Ang II, which is seen by comparing standard diet to the high-salt diet plus losartan. ${ }^{*} P<0.05$ versus high-salt after Bonferroni correction.

ing clearly failed to suppress, and even tended to enhance, Ang IIdependent proximal reabsorption (Figure 1).

An explanation that is unobvious is required to explain why the influence of Ang II over proximal reabsorption was not lessened by dietary salt loading. The explanation that we considered entails dissociating plasma and whole-kidney Ang II from Ang II in the microenvironment of the proximal tubule. If the high-salt diet failed to suppress Ang II in the proximal tubule, this could explain the present micropuncture data, although it would also undo the traditional paradigm for the mechanism of achieving salt balance. To investigate this possibility, we measured Ang II in proximal tubular fluid collected by micropuncture. In contrast to the inhibitory effects of dietary salt on plasma and whole-kidney Ang II, the high-salt diet actually caused the Ang II content of proximal tubular fluid to increase. In other words, dietary salt does affect proximal tubular Ang II, but in a manner that is the reverse of what most physiologists would expect. We do not understand how tubular fluid Ang II came to be increased during dietary salt loading, but the fact that it did could account for 2 unexpected features of the micropuncture data. First, it could fully explain the effects of losartan on proximal reabsorption. Second, it could explain why the range of the TGF response is lessened by losartan but not by dietary salt loading. To summarize this discussion so far, dietary salt exerts a paradoxical effect on Ang II in the proximal tubule such that the pro-reabsorptive influence of Ang II in the proximal tubule is not suppressed by dietary salt loading.

So why might the kidney, in its wisdom, employ an independent Ang II system in the proximal tubule that responds in a contrary way to dietary salt? To answer this question, let us consider how the effects of dietary salt would have differed had all Ang II acting on the tubule been of systemic origin and therefore suppressed by a high-salt diet. This would have the effect of incorporating into the transition from a standard to a high-salt diet those differences that we actually observed between a standard diet without losartan and a high-salt diet with losartan. In other words, without compensation from the tubular Ang II system, increasing dietary salt would have lessened the reactivity of the TGF system, reduced proximal reabsorption, and further increased the delivery of salt to the distal nephron, all without altering the effect of dietary salt on GFR.

There is a mainstream view that, except under extreme circumstances, the kidney prefers to deliver a constant load to the distal nephron and to adjust the salt excretion from there. Our previous experience with early distal micropuncture in normal rats operating over a wide range of salt intakes supports this view (11). The present data are ambiguous in this regard, since we did not sample the tubular fluid far enough downstream to confirm that the impact of increased SNGFR was fully abrogated by increased reabsorption prior to the macula densa. In order to accomplish stable distal salt delivery, proximal reabsorption must be prevented from decreasing when major stimuli to proximal reabsorption (e.g., systemic Ang II and renal sympathetic nerve activity; refs. 22 and 23) decline in response to increased total body salt. A stable distal delivery also hinges on the ability of TGF to filter out fluctuations in SNGFR or tubular function (10), and TGF is only effective when there is Ang II operating in the background (24). The present data reveal that the kidney musters tubular Ang II to offset the decline in proximal reabsorption and TGF responsiveness that would otherwise occur in transition to a high-salt diet. Thereby, proximal tubular Ang II contributes to more stable distal salt delivery.

But if proximal tubular Ang II contributes to more stable distal salt delivery, then it must detract from the overall efficiency of salt homeostasis. This trade-off is inevitable, since by reducing the influence of total body salt over VLP, tubular Ang II downgrades the potential contributions of glomerular filtration and proximal reabsorption to salt homeostasis. It can be proved by logical argument, and without reference to any particular mechanism, that perfect long-term salt balance is inevitable (25). By extension, the impact of salt intake on the steady-state total body salt is completely determined by the shortest interval that can be considered long-term (26). Homeostatic nerves and hormones do not account for the inevitability of salt balance; rather, it transcends them. They merely serve to alter the duration required to restore balance
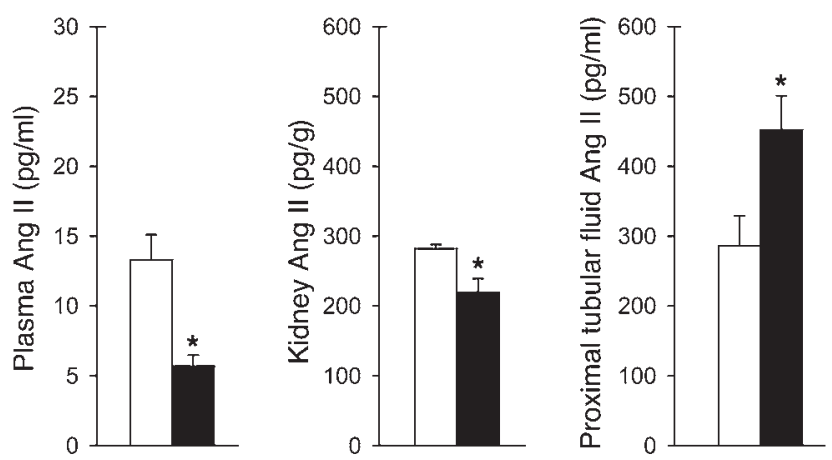

\section{Figure 3}

Ang II in plasma, whole kidneys, and proximal tubular fluid of rats on standard (white bars) or high-salt diet (black bars; $n=4-5$ rats per group; mean $\pm \mathrm{SEM})$. ${ }^{*} P<0.05$ versus standard diet. 
after a change in salt intake, thereby affecting the influence of salt intake on total body salt. Hormones that interact with total body salt through negative feedback shorten this duration, while hormones that interact with total body salt through positive feedback lengthen it. A conspicuous feature of generic angiotensin in physiology is its central role in augmenting the efficiency of salt homeostasis by negative feedback (1). However, the present findings place proximal tubular Ang II and total body salt in a feedback loop with positive gain. Regardless of all other mechanisms involved in salt homeostasis, its presence in a feedback loop with positive gain is sufficient to establish that proximal tubular Ang II detracts from the overall efficiency of salt homeostasis, even though the inescapable requirement for long-term balance implies that gains of all the mechanisms involved sum to a negative number.

While we are the first to our knowledge to point out that increasing dietary salt paradoxically increases tubular Ang II, there is a hint of this phenomenon in the published literature. The proximal tubule is known to contain all the ingredients necessary to make Ang II and it secretes Ang II into the tubular fluid (reviewed in ref. 2). Therefore, Ang II activity in the microenvironment of the proximal tubule need not mirror the activity of the systemic RAS, which varies inversely with dietary salt. Since the kidney scavenges and stores Ang II from the systemic circulation, it has been suggested that changes in kidney Ang II might amplify changes in systemic Ang II (27). On the other hand, maneuvers intended to chronically enhance systemic RAS activity have been reported to cause corresponding increases in whole-kidney Ang II, but not to affect Ang II in the proximal tubular fluid $(28,29)$. On closer inspection of the data in those published reports, they hint at an opposite relationship between systemic and tubular fluid Ang II. The relevant data were published by 1 group of investigators over a period of 10 years and were obtained from normal rats $(30,31)$ and rats manipulated to have high systemic Ang II $(27,28)$. Based on the finding that the concentration of Ang II was typically 50-fold higher in the tubule than in plasma, those authors reasonably chose to ignore lesser differences in the tubular fluid Ang II and maintain that tubular fluid Ang II is effectively the same in normal rats as in rats undergoing maneuvers to increase systemic Ang II (29). With the caveat that the control and experimental data were obtained years apart, tubular fluid Ang II concentrations in those studies appear to be lower in the rats with high systemic Ang II states $(9 \pm 1$ and $5 \pm 1$ nM; refs. 27 and 28$)$ than in normal rats $(13 \pm 2$ and $8 \pm 2 \mathrm{nM}$; refs. 30 and 31 ). The present data confirm that feeding a high-salt diet to suppress the systemic RAS truly does increase the tubular fluid Ang II. Boer et al. made similar findings to ours using acute volume expansion, rather than dietary salt, to dissociate tubular Ang II from the systemic RAS (6).

The present data reveal certain effects of a high-salt diet on the amount of proximal tubular Ang II but do not speak to its origins. Previous reports suggest the existence of an autocrine RAS in the proximal tubule, which secretes abundant angiotensinogen $(4,32)$, and where renin mRNA can be detected by liquid-phase RT-PCR (33). However, the amount of renin present in proximal tubules remains below the level of detection by immunocytochemistry, and the transcript has not been detected by in situ hybridization after RT-PCR, leading others to speculate that the renin signal from liquid-phase RT-PCR represents "illegitimate transcription" and that the proximal tubule likely employs filtered renin to make Ang II (31). In any case, what data there are on proximal tubular renin suggest that the abundance of its transcript is reduced, not augmented, by dietary salt (32). Contrary to the case for proximal tubular renin, the highsalt diet was shown to augment urinary excretion of angiotensinogen fragments in 2 strains of mouse. This angiotensinogen was argued to be of proximal tubular origin (34). Furthermore, the effect of dietary salt on angiotensinogen excretion was dissociated from its effect on the abundance of angiotensinogen mRNA in the kidney (33).

It is likely that tubular and systemic RASs compete for control of the proximal tubule. The present experiments were designed to avoid confounding by salt depletion. It is plausible, or even probable, that the positive effect of dietary salt on tubular angiotensin would not have been apparent had we studied the response to salt depletion, in which case activation of the systemic RAS could have been overwhelming.

The positive influence of dietary salt on tubular Ang II may resolve an apparent contradiction in modern medical practice. Ang II and dietary salt are both impugned in chronic kidney disease. The case for salutary effects of angiotensin blockade is strong, and even brief exposure of tubular epithelial cells to Ang II elicits a marked induction of hundreds of genes for various nuclear factors, hormones, hormone receptors, etc., with consequences that are putatively undesirable (35). Meanwhile, the case for withholding salt from the diet continues to be made (36). But why should dietary salt be harmful if one of its main effects is to suppress the nefarious RAS? The present data undo the contradiction by showing that a high-salt diet exposes the tubule to more Ang II, not less.

\section{Methods}

Overview. All animal experiments were conducted in accord with the NIH Guide for the Care and Use of Laboratory Animals. The protocols were reviewed and approved by the Institutional Animal Care and Use Committee of the VA San Diego Healthcare System. Male Wistar rats were given free access to standard rat chow and water up until the time of the experiments. Those designated to receive the high-salt diet were given $1 \% \mathrm{NaCl}$ in the drinking water for 7 days. This high-salt regimen was previously shown to increase salt intake and excretion by 4 fold (37). The role of Ang II in mediating the effects of dietary salt on proximal tubular function was examined by micropuncture. This was accomplished in 2 series of experiments. In the first series, proximal reabsorption was measured before and during AT1 receptor blockade in rats fed standard or high-salt diets. AT1 receptor blockade was achieved with losartan $(10 \mathrm{mg} / \mathrm{kg}$ i.v. $)$. This dose was confirmed in pilot studies to abolish the hypertensive response to a 5-ng Ang II bolus and to reduce by $80 \%$ the response to a 100-ng Ang II bolus given 1.5 hours later. In the second series of experiments, fluid was collected from free-flowing midproximal tubules of rats fed standard or high-salt diets and assayed for Ang II by radioimmunoassay.

Surgical preparation for micropuncture. Micropuncture was performed under Inactin (100 mg/kg i.p.; Research Biochemicals International) anesthesia according to standard protocols as previously described (38). Animals received Ringer's saline at $2 \mathrm{ml} / \mathrm{h}$ by continuous infusion, with $\left[{ }^{3} \mathrm{H}\right]$ inulin $(80 \mu \mathrm{Ci} / \mathrm{ml})$ added for those experiments requiring a marker of GFR. Animals were allowed 60 minutes to equilibrate prior to beginning micropuncture. SNGFR was measured by $\left[{ }^{3} \mathrm{H}\right]$ inulin clearance in timed collections of tubular fluid made by standard micropuncture.

Assessing proximal reabsorption. To test for primary effects on tubular reabsorption, one must control for differences in load delivered to the tubule by glomerular filtration. To accomplish this, we used TGF as a tool for manipulating SNGFR so that Jprox could be determined as a function of SNGFR in each nephron. Late proximal nephrons were localized on the kidney surface, and an obstructing wax block was inserted immediately upstream of the most downstream-accessible segment. A microperfusion 
pipette with ATF (containing $130 \mathrm{mM} \mathrm{NaCl}, 10 \mathrm{mM} \mathrm{NaHCO}_{3}, 4 \mathrm{mM} \mathrm{KCl}$, $2 \mathrm{mM} \mathrm{CaCl}_{2}, 7.5 \mathrm{mM}$ urea, $0.1 \% \mathrm{FD} \& \mathrm{C}$ Green 3, $\mathrm{pH}$ 7.4.) was inserted downstream of the wax block to perfuse Henle's loop with the aid of a Hampel nanoliter pump (Department of Pharmacology, University of Tübingen). Controlled perfusion of Henle's loop was performed in order to activate TGF, thereby causing SNGFR to change. While perfusing Henle's loop to manipulate SNGFR, timed collections of tubular fluid were made upstream of the wax block in order to measure SNGFR and VLP. Collections were made from each nephron during maximal TGF activation (loop of Henle microperfusion at $38 \mathrm{nl} / \mathrm{min}$ ) and minimal TGF activation (loop of Henle microperfusion at $0 \mathrm{nl} / \mathrm{min}$ ). Nephrons were vented upstream from the wax block before each collection to prevent pressure from building up in the proximal tubule. Suction was applied momentarily to break surface tension at the pipette tip. Otherwise, tubular fluid was allowed to enter the pipette spontaneously without suction.

Two minutes were allowed for equilibration prior to each collection, and each collection was for 3 minutes. Tubular fluid samples were assayed for volume by transfer to a constant-bore glass capillary and then counted for radioactivity to determine SNGFR. Data from these paired collections were exploited to characterize proximal reabsorption as a function of SNGFR by linear interpolation. Primary effects of dietary salt and losartan were defined by differences in these functions. A detailed justification for this method to test for effects on proximal reabsorption has been previously published (12).

Ang II content of proximal tubular fluid. Ten-minute collections were made by micropuncture from midproximal tubular sites of rats fed standard or high-salt diets. Midproximal sites were identified as being at least 5 proximal tubular loops upstream from the last proximal tubular surface segment. According to the protocol of Braam, Navar, and colleagues (30), samples were measured for volume, then placed in $1 \mathrm{ml}$ of cold methanol and stored at $-20^{\circ} \mathrm{C}$ until radioimmunoassay. Multiple collections were performed to collect a total of about $2 \mu \mathrm{l}$ per animal. On the day of the assay, samples were dried to remove the methanol, and the lyophylisate was subjected to radioimmunoassay (see below).

Ang II content of plasma and whole kidney. In 2 additional sets of rats treated exactly as those described above, plasma Ang II and kidney Ang II were measured by radioimmunoassay according to a protocol previously used in this laboratory $(20,39)$.

Plasma processing. Animals were anesthetized with Inactin $(100 \mathrm{mg} / \mathrm{kg}$ i.p.), and, following an abdominal incision, the abdominal aorta was punctured and $1.5 \mathrm{ml}$ of blood was collected in a syringe containing $20 \mu \mathrm{LDTA}$ $(0.16 \mathrm{M})$ and $10 \mu \mathrm{l}$ converting enzyme inhibitor $(0.1 \mathrm{mM})$. Samples were spun $(2,000 \mathrm{~g}$ for 10 minutes $)$ at $4^{\circ} \mathrm{C}$, and plasma was stored at $-70^{\circ} \mathrm{C}$ until processed. Plasma was extracted using a Bondelut $\mathrm{C}_{18}$ column previously washed with methanol and triethylamine formic acid. The column was rinsed with triethylamine formic acid buffer, and Ang II was eluted off with acetonitrile triethylamine formic acid (70:30 dilution), lyophilized on a SpeedVac centrifuge (Savant Instruments Inc.) overnight, and kept at $-20^{\circ} \mathrm{C}$ until assayed. This extraction procedure yielded $92 \%$ recovery of Ang II.

Kidney processing. Animals were anesthetized with Inactin (100 mg/kg i.p.), the abdomen was opened, and both kidneys were rapidly excised, flash-frozen in liquid nitrogen, and stored at $-70^{\circ} \mathrm{C}$ until further processing. Individual whole kidneys were homogenized (Polytron, 10 seconds at 6 setting;
Brinkman Instruments Inc.) in $2 \mathrm{ml}$ radioimmunoassay-BSA (RIA-BSA) $0.25 \%$ buffer, added to $9 \mathrm{ml}$ of homogenizing medium (1 $\mathrm{N}$ glacial acetic, $0.02 \mathrm{~N}$ hydrochloric acid, and $0.1 \%$ 2-mercaptoethanol), and heated to $90^{\circ} \mathrm{C}$ for 10 minutes, then centrifuged at $30,000 \mathrm{~g}$ for 20 minutes. The supernatant $\mathrm{S}_{1}$ was removed, and the pellet was resuspended in $4.5 \mathrm{ml}$ of homogenizing medium and centrifuged at $30,000 \mathrm{~g}$ for 20 minutes. The resulting supernatant was combined with $S_{1}$ and lyophilized on a SpeedVac centrifuge overnight. The resulting lyophilisate was resuspended in 2 ml RIA-BSA $0.25 \%$ buffer, and $500 \mu \mathrm{l}$ was extracted using a Bondelut $\mathrm{C}_{18}$ column as described above for plasma. This combined procedure yielded 59\% recovery of Ang II.

Ang II radioimmunoassay. This assay has been reported previously and validated by HPLC (39). Briefly, lyophilisates of plasma, kidneys, or tubular fluid were suspended in $500 \mu \mathrm{l}$ (200 $\mu \mathrm{l}$ for tubular fluid samples) of RIABSA buffer, and $200 \mu \mathrm{l}$ of this was added to $100 \mu \mathrm{l}$ of specific rabbit Ang II antibody diluted 1:62,500 (Ab 127, provided by W. Vale, Salk Institute, La Jolla, California, USA). Cross-reactivity of this Ang II antibody with Ang I is $0.33 \%$ and with Ang III is $68 \%$. After incubating for 2 days at $4{ }^{\circ} \mathrm{C}, 100$ $\mu 1^{125}$ I-AII (6,000 cpm; New England Nuclear) was added to each tube and incubated again at $4{ }^{\circ} \mathrm{C}$ overnight. Ang II bound to antibody was separated from unbound Ang II using normal rabbit serum diluted 1:200 (100 $\mu \mathrm{l})$ and goat anti-rabbit IgG diluted 1:40 (100 $\mu \mathrm{l})$, and $500 \mu \mathrm{l} 10 \%$ polyethylene glycol in RIA-BSA buffer was added to each tube. Tubes were incubated at $4^{\circ} \mathrm{C}$ for 2 hours and then spun for 30 minutes, after which the supernatant was decanted and the pellet was counted in a $\gamma$ counter.

Statistics. Statistical analysis was by 1 - or 2-way analysis variance or covariance as appropriate, using commercial software (SYSTAT version 6.0.1; SPSS). Body weight, which did not differ between groups, was included as a covariate since it accounted for much of the within-group variability in SNGFR. Micropuncture collections with tubular fluid to plasma inulin ratio more than 1.8 standard deviations from the mean were cast as outliers to avoid including inadvertent distal tubule collections. As is customary for micropuncture data, individual collections were treated as separate data points. To test for primary differences in proximal reabsorption, values for Jprox were derived as a function of SNGFR by linear interpolation of the group means \pm SEM measured with and without TGF activation. Intergroup comparisons of proximal reabsorption at a given SNGFR were made by 2 -tailed Student's $t$ test of values derived for given SNGFR by linear interpolation as previously described (12). Statistical significance was assigned for $P<0.05$.

\section{Acknowledgments}

This work was supported by the Office of Research and Development of the Veterans Health Administration and by NIH grants DK56248 and DK28602.

Received for publication June 28, 2005, and accepted in revised form February 7, 2006.

Address correspondence to: Scott Thomson, Department of Medicine, Division of Nephrology - Hypertension, University of California, San Diego, and VA San Diego Healthcare System, 3350 La Jolla Village Drive 9151, San Diego, California 92161, USA. Phone: (858) 552-7528; Fax: (858) 642-1438; E-mail: sthomson@ucsd.edu.
1. Guyton, A.C., Hall, J.E., Coleman, T.G., and Man-
ning, R.D. 1990. The dominant role of the kidneys
in long-term regulation of arterial pressure in nor-
mal and hypertensive states. In Hypertension patho-
physiology, diagnosis, and management. J.H. Laragh
and B.M. Brenner, editors. Raven Press. New York,
New York, USA. 1029-1052.
2. Navar, L.G., Imig, J.D., Zou, L., and Wang, C.T.
1997. Intrarenal production of angiotensin II. Semin. Nephrol. 17:412-422.

3. Vos, P.F., Boer, P., Braam, B., and Koomans, H.A. 1994. The origin of urinary angiotensins in humans. J. Am. Soc. Nephrol. 5:215-223.

4. Rohrwasser, A., et al. 1999. Elements of a paracrine tubular renin-angiotensin system along the entire nephron. Hypertension. 34:1265-1274.
5. Gociman, B., et al. 2004. Expression of angiotensinogen in proximal tubule as a function of glomerular filtration rate. Kidney Int. 65:2153-2160.

6. Boer, W.H., Braam, B., Fransen, R., Boer, P., and Koomans, H.A. 1997. Effects of reduced renal perfusion pressure and acute volume expansion on proximal tubule and whole kidney angiotensin II content in the rat. Kidney Int. 51:44-49. 
7. Steiner, R.W., Tucker, B.J., and Blantz, R.C. 1979. Glomerular hemodynamics in rats with chronic sodium depletion. Effect of saralasin. J. Clin. Invest. 64:503-512.

8. Pelayo, J.C., Ziegler, M.G., and Blantz, R.C. 1984. Angiotensin II in adrenergic-induced alterations in glomerular hemodynamics. Am. J. Physiol. 247:F799-F807.

9. Vallon, V., Peterson, O.W., Gabbai, F.B., Blantz, R.C., and Thomson, S.C. 1995. Interactive control of renal function by alpha 2 -adrenergic system and nitric oxide:role of angiotensin II. J. Cardiovasc. Pharmacol. 26:916-922.

10. Thomson, S.C., and Blantz, R.C. 1993. Homeostatic efficiency of tubuloglomerular feedback in hydropenia, euvolemia, and acute volume expansion. Am. J. Physiol. 264:F930-F936.

11. Vallon, V., et al. 2002. Salt-sensitivity of proximal reabsorption alters macula densa salt and explains the paradoxical effect of dietary salt on glomerular filtration rate in diabetes mellitus. J. Am. Soc. Nephrol. 13:1865-1871.

12. Thomson, S.C., et al. 2001. Ornithine decarboxylase, kidney size, and the tubular hypothesis of glomerular hyperfiltration in experimental diabetes. J. Clin. Invest. 107:217-224.

13. Schnermann, J., and Briggs, J.P. 1990. Effect of angiotensin and other pressor agents on tubuloglomerular feedback responses. Kidney Int. Suppl. 30:S77-S80.

14. Mitchell, K.D., and Navar, L.G. 1988. Enhanced tubuloglomerular feedback during peritubular infusions of angiotensins I and II. Am. J. Physiol. 255:F383-F390.

15. Shultz, P.J., and Tolins, J.P. 1993. Adaptation to increased dietary salt intake in the rat. Role of endogenous nitric oxide. J. Clin. Invest. 91:642-650.

16. Vallon, V., and Thomson, S.C. 1995. Inhibition of local nitric oxide synthase increases the homeostatic efficiency of tubuloglomerular feedback. Am.J. Physiol. 38:F892-F899.

17. Welch, W.J., and Wilcox, C.S. 1997. Role of nitric oxide in tubuloglomerular feedback: effects of dietary salt.
Clin. Exp. Pharmacol. Physiol. 24:582-586.

18. Vallon, V., et al. 2001. Feedback control of glomerular vascular tone in neuronal nitric oxide synthase knockout mice. J. Am. Soc. Nephrol. 12:1599-1606.

19. Ushiogi, Y., and Haberle, D.A. 1991. Hyperreactivity of tubuloglomerular feedback in chronically salt-loaded spontaneous hypertensive rats. Kidney Int. Suppl. 32:S142-S147.

20. Vallon, V., Wead, L.M., and Blantz, R.C. 1995. Renal hemodynamics and plasma and kidney angioten$\sin$ II in established diabetes mellitus in rats: effect of sodium and salt restriction. J. Am. Soc. Nephrol. 5:1761-1767.

21. Ingert, C., Grima, M., Coquard, C., Barthelmebs, M., and Imbs, J.L. 2002. Effects of dietary salt changes on renal renin-angiotensin system in rats. Am. J. Physiol. Renal Physiol. 283:F995-F1002.

22. Bello-Reuss, E., Colindres, R.E., Pastoriza-Munoz, E., Mueller, R.A., and Gottschalk, C.W. 1975. Effects of acute unilateral renal denervation in the rat. J. Clin. Invest. 56:208-217.

23. Lohmeier, T.E., Hildebrandt, D.A., and Hood, W.A. 1999. Renal nerves promote sodium excretion during long-term increases in salt intake. Hypertension. 33:487-492.

24. Schnermann, J.B., et al. 1997. Absence of tubuloglomerular feedback responses in AT1A receptordeficient mice. Am. J. Physiol. 273:F315-F320.

25. Guyton, A.C. 1990. The surprising kidney-fluid mechanism for pressure control--its infinite gain! Hypertension. 16:725-730.

26. Walser, M. 1985. Phenomenological analysis of electrolyte and water homeostasis. In The kidney: physiology and pathophysiology. D.W. Seldin and G. Giebisch, editors. Raven Press. New York, New York, USA. 3-13.

27. Navar, L.G., and Harrison-Bernard, L.M. 2000 Intrarenal angiotensin II augmentation in angiotensin II dependent hypertension. Hypertens. Res. 23:291-301.

28. Cervenka, L., Wang, C.T., Mitchell, K.D., and Navar, L.G. 1999. Proximal tubular angiotensin II levels and renal functional responses to AT1 receptor blockade in nonclipped kidneys of Goldblatt hypertensive rats. Hypertension. 33:102-107.

29. Wang, C.T., Navar, L.G., and Mitchell, K.D. 2003. Proximal tubular fluid angiotensin II levels in angiotensin II-induced hypertensive rats. J. Hypertens. 21:353-360.

30. Braam, B., Mitchell, K.D., Fox, J., and Navar, L.G. 1993. Proximal tubular secretion of angiotensin II in rats. Am. J. Physiol. 264:F891-F898.

31. Navar, L.G., Lewis, L., Hymel, A., Braam, B., and Mitchell, K.D. 1994. Tubular fluid concentrations and kidney contents of angiotensins I and II in anesthetized rats. J. Am. Soc. Nephrol. 5:1153-1158.

32. Navar, L.G., Lewis, L., Hymel, A., and Mitchell, K.D. 1996. Proximal tubular fluid levels of angiotensinogen in anesthetized rats. FASEB J. 10:A22.

33. Tank, J.E., Henrich, W.L., and Moe, O.W. 1997. Regulation of glomerular and proximal tubule renin mRNA by chronic changes in dietary $\mathrm{NaCl}$. Am. J. Physiol. 273:F892-F898.

34. Lantelme, P., et al. 2002. Effects of dietary sodium and genetic background on angiotensinogen and Renin in mouse. Hypertension. 39:1007-1014.

35. Braam, B., et al. 2003. Human proximal tubular cell responses to angiotensin II analyzed using DNA microarray. Eur. J. Pharmacol. 464:87-94.

36. Panel on Dietary Reference Intakes for Electrolytes and Water, Standing Committee on the Scientific Evaluation of Dietary Reference Intakes, Food and Nutrition Board. 2004. Dietary Reference Intakes for Water, Potassium, Sodium, Chloride, and Sulfate. National Academies Press. Washington, DC, USA. 617 pp.

37. Vallon, V., et al. 1997. Effect of chronic salt loading on kidney function in early and established diabetes mellitus in rats. J. Lab. Clin. Med. 130:76-82.

38. Blantz, R.C., and Tucker, B.J. 1978. Measurements of glomerular dynamics. In Methods in pharmacology. M. Martinez-Maldonado, editor. Plenum. New York, New York, USA. 141-163.

39. De Nicola, L., Thomson, S.C., Wead, L.M., Brown, M.R., and Gabbai, F.B. 1993. Arginine feeding modifies cyclosporine nephrotoxicity in rats. J. Clin. Invest. 92:1859-1865. 